\title{
An Electrophysiological Approach to the Evaluation of Regional Sympathetic Dysfunction: A Proposed Classification
}

\author{
David R. Longmire, MD
}

Background: The importance to physicians of maintaining a level of understanding of illnesses and their treatment continues to reveal itself in a most striking fashion when it comes to the progressive interest recently directed to disorders of the autonomic nervous system (ANS). In particular, the relevance to pain practitioners of disease states which directly involve the sympathetic portion of the ANS has increased markedly following the international renaming of reflex sympathetic dystrophy (RSD) and causalgia to complex regional pain syndrome (CRPS) Type I and Type II respectively, as well as sympathetically maintained pain (SMP).

Subsequently it has become better understood that many other forms of neuro- pathic pain also demonstrate local abnormalities of the sympathetic nervous supply to the skin within the painful territory, thereby increasing the diagnostic value of these (often subtle) cutaneous clinical signs.

Objectives: The objectives of this presentation include (a) a concise review of laboratory tests that are currently used in the evaluation of the autonomic nervous system, (b) a discussion of those procedures that were developed for the assessment of sympathetic sudomotor function, (c) a review of the anatomic pathways subserving those electrophysiological methods for sudomotor testing, and (d) the current diagnostic classification for regional abnormalities of sympathetic sudomotor dysfunction.
Methods: Methods used in the preparation of this article have included a review of (a) historic clinical and laboratory articles (or translations thereof) regarding the medical importance of disorders of the autonomic nervous system, dating back to more than 155 years ago (b) anatomic and electrophysiological basis for electroneurodiagnostic sudomotor testing, and (c) the author's proposal for a diagnostic classification of regional sympathetic sudomotor dysfunction.

Key words: Autonomic, sympathetic, sudomotor, complex regional pain syndrome, sympathetically maintained pain, selective tissue conductance, Epi-Scan, neuropathic pain, Longmire classification of regional sympathetic dysfunction
Four decades ago the development of modern methods for studies of the autonomic nervous system seemed to represent a neglected pathway within the field of clinical neurophysiological research. However, with the advent of new or expanded laboratory proce-

From: University of Alabama School of Medicine-Huntsville Program, Huntsville, Alabama Address Correspondence:

David R. Longmire, MD

Clinical Associate Professor

Department of Internal Medicine

University of Alabama School of Medicine-Huntsville Program

Huntsville, AL 35653

E-mail: longmire@hiwaay.net

Disclaimer: There was no external funding in the preparation of this manuscript.

Conflict of Interest: All early developmental research regarding one of the methods for sympathetic sudomotor assessment described in this review, i.e. Selective Tissue Conductance (STC) Technology, was performed by the author and W.E. Woodley, M Eng. However, all development leading to the current Epi-Scan P10o STC device and computer analysis system has been performed by EDX Epi-Scan Corporation of Huntsville, AL. Neither the author of this review nor W.E. Woodley, are employees of, or shareholders in, EDX Epi-Scan Corporation.

Manuscript received on: 10/06/2005

Revision submitted on: 12/21/2005

Accepted for publication on: 12/21/2005 dures, medical disorders such as dysautonomia, peripheral or autonomic neuropathy, and sympathetic dysfunction associated with complex regional pain syndromes can now be assessed objectively and quantitatively.

The purpose of this monograph is to provide a brief review of relevant tests of autonomic function, with specific attention to noninvasive electrophysiological procedures by which sympathetic sudomotor disorders may be evaluated objectively and quantitatively

\section{LABORATORY AsSESSMENT OF \\ AUtONOMIC Function}

Current approaches to the clinical assessment of the autonomic nervous system generally involve laboratory procedures which have been designed to test one or more of the following subsystems (1):

Parasympathetic cardiovagal innervation: This evaluation (CPT 95921), is performed predominantly by recording two or more of the following subtests, such as the heart rate response to deep breathing (with recorded R-R interval), the Valsalva ratio, and 30:15 ratio.
Sympathetic vasomotor (adrenergic) innervation: This procedure (CPT 95922) measures beat to beat blood pressure and R-R interval changes during the Valsalva maneuver and during at least 5 minutes of passive tilt.

Sympathetic sudomotor (adrenergic / cholinergic) innervation: This type of autonomic test (CPT 95923) involves the application of procedures designed to evaluate sympathetic nerve pathways that control regional changes in perspiration. These are known as sudomotor fibers (sudor, Latin: sweat; and motor, Latin: motion). Several laboratory methods have been developed for the assessment of sympathetic sudomotor function (2) in animal and human subjects, confirming longstanding but progressive interest in this aspect of autonomic testing. Consequently, the theme of this clinical monograph is directed primarily to the discussion of the application of certain sympathetic sudomotor tests in medical practice.

\section{Physiological Basis of Sympathetic Sudomotor Evaluation}

One of the essential roles of the sympathetic portion of the autonomic ner- 
vous system is the maintenance of a relatively narrow body temperature range in which chemical reactions can occur optimally within cells. This effect, known as thermoregulation, is based upon (a) the constant central monitoring of temperature-related sensory nerve impulses generated distally in the body, (b) the processing thereof within cerebral (e.g. limbic, hypothalamic) autonomic centers, from whence (c) corrective or adaptive efferent supraspinal, spinal and peripheral impulses are initiated and directed to those visceral and cutaneous effectors which can, through their individually specialized functions, return internal temperature levels to a balanced state.

While there is evidence for input from various portions of the cortex and limbic system into the hypothalamus, it is in the preoptic area of this latter structure where central thermoregulatory control begins. From there, efferent nerve fibers descend ipsilaterally and remain uncrossed within the brainstem, terminating in the intermediolateral (IML) fasciculus of the spinal cord.

Within the IML horn of the spinal cord gray matter a separate neuronal population exists known as sympathetic preganglionic neurons. The axons of these form preganglionic fibers, which leave the spinal cord through, or along with, the anterior roots.

Shortly thereafter, these fibers separate from the roots and proceed toward the paravertebral sympathetic chain. Their myelination gives rise to their color and thus the segments of these pathways between the root and the chain ganglia are known as the white rami communicantes. After synapsing with those neurons in the respective ganglia that are destined to become part of the final common sudomotor path, the postganglionic class $C$ fibers exit and, due to their unmyelinated state, are known as the gray rami communicantes. They return to join the anterior root and subsequently the main (mixed) nerve root that passes through bony canals in the spinal column.

\section{Sympathetic Effector Systems}

These fibers continue to travel in parallel with other efferent axons until they branch off to supply the effector organs in their respective cutaneous regions. From there they provide control of the three specialized sets of end organs which contribute to the control of the body's re- action to changes in internal and external temperature.

The first of these end organs are pilomotor effectors which consist of small specialized muscles at the base of each hair root. When activated, they pull the hair shaft upward at an angle away from the surface of the body, allowing air to be trapped long enough to improve insulating effect. When no hair exists, these effectors still may contract, leaving a small mound of skin at each empty follicle, giving the appearance of goose bumps or goose flesh. Pilomotor fibers are least applicable to the quantitative assessment of sympathetic dysfunction and are most difficult to measure in human subjects.

Vasomotor fibers, a second type of sympathetic efferents, help regulate regional blood flow through their neural control over the selective constriction of arterioles. When such changes occur in the skin, the amount of flow permits the transfer or conservation of heat relative to the temperature of the surrounding air.

Sudomotor effectors, a third set of sympathetic effectors, consist of the sweat glands which contribute to body cooling through the production and expulsion of perspiration. This in turn releases heat from the body surface through the evaporation of sweat. It is to the identification and localization of lesions of the autonomic nervous system that laboratory methods of sympathetic sudomotor testing have been most successfully applied.

\section{Clinical Tests of Sudomotor Function}

At present there are four general categories of tests used for sudomotor evaluation: iontophoretic, sweat imprint, colorimetric and electrophysiologic (1). While published data regarding such tests appear to suggest that each method is novel, many of the current procedures are based upon earlier research, much of which was performed over half a century ago $(2,3)$.

\section{Iontophoretic: Quantitative Sudomotor Axon Reflex Test}

One system of measuring sweat output from discrete areas of skin has been developed by Low and colleagues (4) for the assessment of autonomic neuropathy. This method, known as the Quantitative Sudomotor Axon Reflex Test (QSART), provides a precisely controlled microenvironment at individual cutaneous sites within which sweat latencies and response amplitudes to iontophoretic stimulation can be calculated.

The correct application of QSART to single-site assessment of cutaneous autonomic dysfunction requires a combination of interrelated laboratory instruments, including a sudorometer, a constant current generator, and a sweat cell. All of these components are essential for the acquisition of the sensitive and physiologically detailed data which are expected from this test

The QSART transducer is applied to a predetermined site on the surface of human skin and its mechanical stability is confirmed before testing is attempted. Dry (dehumidified) nitrogen is passed through a flow regulator at a controlled rate into a temperature-controlled humidity sensor. The nitrogen, of known flow rate and humidity, passes through the sweat cell and causes total evaporation of the sweat produced within the boundaries of its sealed chambers. The nitrogen, made moist by the sweat expelled, is returned to the humidifier sensor where the relative humidity change is detected and calculated using a dedicated computer system. The design of the sweat cell includes a well which is divided into three compartments: a recording compartment, an air gap compartment, and an acetylcholine (ACh) well. The basic pre-test procedure consists of control measurements of resting sweat output (RSO) before the introduction of a $10 \%$ solution of acetylcholine into the ACh well. Next, the ACh solution is electrophoresed through the skin. Shortly after ACh iontophoresis, the sweat response is measured in terms of latency, volume, and area under the curve produced by the measured values. While this test is based upon solid physiological principles, there are some instrumental and financial limitations in terms of developing a QSART system that can provide simultaneous evaluation of multiple areas of skin. As well, there is some modest discomfort which presents itself during the test as a stinging or burning sensation beneath each transducer.

\section{Silastic Sweat Imprint Method}

One detailed method used to document sweat gland and sweat pore patterns involves the application of certain types of glue, plastic, or latex material to the surface of the skin in a manner by which the liquid agent would seep down into the pores. When dry, the rubbery imprint can be peeled of the skin, resulting in 
a negative impression of the ducts and their exit foramina. The most detailed procedure for this purpose appears to be the silastic mold technique developed by Kennedy and Navarro (5). By applying liquid silastic to small regions of skin and permitting it to dry, a cast of the sweat pore and the limbus of the sweat duct can be obtained. As it is removed from the skin, the three dimensional product can be examined using a wide variety of methods, including scanning electron microscopy.

This test has a much greater precision than several of its predecessors. However, the application of the silastic material to multiple sites, and the amount of time required for the quantitative analysis of each sample, together may make the method excessively complex for clinical testing of large areas.

\section{Colorimetric Assessment of Regional Perspiration: The Thermoregulatory Sweat Test}

Most colorimetric tests of sudomotor function are based on the principle that certain combinations of liquid and powdered chemicals have the ability to change color when they become wet. The underlying hypothesis for their use in sudomotor testing is that such chemicals, when applied to dry human skin, retain their original pale color until the body is sufficiently heated enough to produce increased thermoregulatory sweating. When such a sweat response is evoked, the chemical powders or dyes become wet, and consequently their colors become much darker wherever sweating has occurred. Conversely, the chemical colors do not change in regions of cutaneous sympathetic denervation (i.e., in areas where no normal sweating can be found).

One early form of the Thermoregulatory Sweat Test (TST) was first used by Minor (6) over 70 years ago and consisted of a mixture of iodine, castor oil, and diluted alcohol. This was used to paint the skin over the territory of interest until the skin was yellow. At that point, starch powder was sprinkled over the dried skin until the appearance was white. As long as there was little perspiration, the powder did not change in color. When sweating was induced by heating the subject, sweat droplets became visible as dark spots on a homogeneous white background. As sweating increased, the spots on the skin grew in size until the entire territory of the mixture became dark.
Further chemical materials were introduced for the same purpose, a common example of which included quinizarin as used by Guttmann (7). However, the most modern variation of this method appears to be that recommended by Fealey (8) in which the chemicals used are alizarin red mixed with corn starch and sodium carbonate. The powder mixture is light orange when dry and purple when wet. Patients who are referred for TST are prepared in a cooler environment, following which they are placed in a heating chamber until the desired amount of sweating is attained.

Unfortunately, all colorimetric techniques are associated with a certain degree of inconvenience, complexity, and untidiness during the application and removal of the chemicals. As well, the interpretation of regional TST abnormalities is extremely difficult in certain painful disorders since spontaneous local hyperhidrosis causes discoloration even before body heating.

Electrophysiological Assessment of the Skin: Sympathetic Skin Response and Related Electrodermal Activity

Since the middle of the $19^{\text {th }}$ century, physicians have accepted the clinical application of electrophysiological methods to the study of normal and abnormal function in the human nervous system (9). The subsequent invention of oscillographic ink; or heat writers, permitted the creation of hard copy recordings of bioelectric activity generated in many different body systems. This created subspecialties within the field of electrophysiology, the names of which were created by placing the prefix electro before, and the suffix ography after, any root word indicating the body system being tested.

As a consequence, the term electrodermography (EDG) (10) is currently used to encompass the wide range of electrophysiological measurements related to skin that were previously known as the galvanic skin response (GSR) (11). While the original term GSR was intended to be used solely to represent one specific electrical skin phenomenon, it eventually became widely applied to all forms of electrodermal measurement.

\section{Active Electrodermographic Pheno- mena: Sympathetic Skin Response}

When variations occur in the production or expulsion of sweat, there are measurable physiological changes in the skin which are classified from an electrical perspective as being either predominantly active (endosomatic) or passive (exosomatic).

Active electrical changes are the result of the volume averaging of transmembrane electrical potential differences generated within skin cells, and hence are classified as endosomatic. Those which are reasonably steady or slow to change in a resting state, are collectively known as the skin potential level. Large fluctuations in skin potential level which occur after the presentation of an acute stimulus are generally categorized as skin potential responses or, historically, as Tarchanoff's Effect $(12,13)$.

In 1924, Richter (14) had one of the earliest reported opportunities to study the effects of a structural lesion of the sympathetic chain on the evoked GSR. In one patient with a painful lesion of the cervical sympathetic chain, he noted a marked difference in sweating between symptomatic and asymptomatic sides of the face, neck, upper chest, arms, and hands. With reference to the upper limbs of this patient, he attempted to obtain some objective measurement of this pathological state by using contemporary GSR methods and instrumentation. His detailed recordings of phasic resistance changes occurring at rest and after sensory stimulation revealed a loss of the expected GSR of the hand ipsilateral to the lesion. Responses recorded from the asymptomatic arm and hand remained consistent with those of normal controls. The clinical correlation of this type of sympathetic abnormality found subsequent uses by anesthesiologists in the assessment of the effects of regional sympathetic neurolytic blockade (15-17) albeit by different names. Current terminology (1) for use of the skin potential response in medical practice has followed the changes proposed by Shahani et al $(18,19)$ and has adopted the term sympathetic skin response. The Shahani method has been used in the assessment of various forms of peripheral autonomic neuropathy and sympathetic dysfunction.

\section{Passive Electrodermographic Pheno- mena: Sympathetic Skin Resistance and Selective Tissue Conductance}

The electrical characteristics of skin which reflect the function of the body surface by allowing or opposing the passage 
of direct electrical current, phenomena known respectively as conductance or resistance, are considered passive. Since their assessment is based upon tissue reactions to externally applied test current, these types of electrodermal measurements are also classified as exosomatic.

The medical application of exosomatic electrodermographic (EDG) measurement is based upon one main principle: hypohidrotic or anhidrotic skin creates more resistance to the flow of externally applied electrical current than does euhidrotic or hyperhidrotic skin.

In clinical practice this resistance occurs most frequently when the sympathetic nerve supply to the skin is interrupted, skin moisture decreases, and skin electrical resistance rises within the cutaneous territory of the damaged nerve. This effect was first reported as early as 1879 by Vigouroux (20), senior electrophysiologist at Charcot's department of neurology at the Salpetriere in Paris. He was among the first to discover that damage to distal segments of a single peripheral nerve increased the electrical resistance of the region of skin originally served by that nerve.

Unfortunately the potential clinical applicability of passive electrical measurement of this type was not to be acknowledged until nearly half a century later when Richter abandoned his use of the evoked GSR. He, his colleagues, and students embarked on more extensive studies using clinical correlation of electrical skin resistance levels in patients with various pathological or surgical lesions of individual peripheral nerves, nerve roots, sympathetic ganglia, or spinal cord. As such, Richter and his colleagues (21-31) were able to map the sympathetic innervation to the entire body surface, an effort which remains historic in basic and clinical neurobiology.

\section{Selective Tissue Conductance}

Despite the extensive use of the skin resistance method, there were a few inherent technical concerns regarding methodology and clinical applicability. Therefore, in order to enhance the quantitative aspects of Richter's method while improving safety and efficacy, in 1963 the author initiated a series of projects to that end, and in 1973 he and William E. Woodley began collaborating on a series of projects that eventually led to the establishment of Selective Tissue Conductance (STC) tech- nology, as a painless, noninvasive method, classified by HHS/FDA $(32,34)$ for evaluating regional sympathetic sudomotor dysfunction.

\section{Conductance versus Resistance}

The first step in the development of STC instrumentation was the decision to use skin conductance, instead of skin resistance, as the measurement of choice. This selection was based upon the fact that levels of conductance are directly proportional to the activity of sympathetic sudomotor efferent fibers, whereas resistance levels are inversely related to sympathetic outflow. Early investigations on the variability of skin electrical responses created by changing parameters of stimulation indicated that the measurement of conductance levels, not responses, would provide more stable results.

Although this type of instrumentation was originally intended for the measurement of human skin conductance, there have been suggested applications of electrical assessment of other types of tissue (e.g. in the fields of marine biology and plant physiology), as well as assessment of the condition of necrotic muscle during surgery. Therefore, the term Selective Tissue Conductance was recommended as being more appropriate for the broad range of biological materials that could be evaluated with this method.

\section{Spatial Selectivity: The Elimination of Transcorporeal Currents}

Early methods of measuring skin resistance often consisted of passing an electrical test current between a static reference electrode and a roving or exploring electrode that was moved over the areas of the skin to be assessed. If it happened that the reference and roving electrodes were placed on opposite sides of the body, then electrical flow could traverse the body creating transcorporeal currents. If this current path flowed through electrically sensitive organs such as the heart, then at least potential, if not actual, risks of arrhythmia could be increased.

This effect was eliminated in 1982 when Longmire and Woodley developed a bipolar concentric electrode that could simply be held against the unprepared skin surface, without the need for electrode gels or creams. Its design consists of a central disk separated from an outer ring by an insulator. This allows a restricted passage of the test current between the center contact and the outer ring electrode through very superficial layers of the skin by volume conduction, with no possibility of transcorporeal current. This aspect of Selective Tissue Conductance technology is known as spatial selectivity.

\section{Temporal Selectivity: The Avoidance of Iontophoretic Effect}

The usual measurement of conductance in biological tissue with direct current test signals is associated with progressively increasing values based upon the duration of application of current. This is known as the iontophoretic effect, and initially represented a potential source of variability of measurement. However, by making conductance measurements early in the application of current, using a method known as temporal selectivity, it was possible to eliminate this effect.

\section{Selective Tissue Conductance: Operational Definition}

By combining these elements of conductance, spatial selectivity and temporal selectivity, the operational definition for Selective Tissue Conductance has been established as "...the relative ability of biological tissue to conduct a weak (DC) electrical signal, which is applied for a selected period of time to a selected, limited and restricted surface area of that tissue..." $(32,33)$

\section{Units of Measurement}

Until the introduction of a new set of units of physical measurement in the 1970s by the Système Internationale (SI), the unit for electrical conductivity was the Mho, later changed to Siemens in honor of the contributions of the German scientist who extensively researched the electrical properties of conducting and insulating materials. The normal range of conductivity of human skin is wide, but absolute levels are generally very low, often running in the micro- or nano-Siemens levels. Since the conductivity of skin is dependent upon the surface area of the electrodes, the standard unit of measurement for Selective Tissue Conductance is nanoSiemens per square centimeter $\left(\mathrm{nS} / \mathrm{cm}^{2}\right)$.

\section{Standardization of Selective Tissue Conductance Methods I: MeAsurement}

The application of STC technology to the clinical assessment of regional sudomotor dysfunction has been established for 
over 25 years and in over 4,600 tests within the United States. Within the last five years, many advances in Epi-Scan STC instrumentation, notably the computer-based P100 system of M.C. Patel, PhD (Personal communication, 1999-present) have led to new methods of examination, data collection, quantitative analysis, and reporting. This has included the standardization of each component of a comprehensive examination system for this particular type of sympathetic sudomotor evaluation.

\section{Single Site Measurements}

One of the earliest methods of STC assessment consisted of measurements made at a single predetermined site, known as Type A reference studies (34). These were most often located over the midline of the forehead, chest, abdomen, or spine. Single site data of this type shared the same spatial limitations as QSART and the Silastic Sweat Imprint Test. However, the strength of this technique was demonstrated when single site measurements were repeated at equal intervals over time during and after medical treatment or the induction of hyperhidrosis through gustatory or noxious stimulation, such as the single digit cold pressor method. It may also be used to assess the dynamic STC range over certain areas of skin. At present it is most often used as a bioelectric test technique before and after performing a comprehensive evaluation of sympathetic sudomotor function, such as the Patel Grid.

\section{Multiple Site Measurements}

Comparative (Symptomatic /

Asymptomatic) Studies

Originally known as Type B studies, these methods consisted of comparing the STC values of multiple sites distributed over anatomic landmarks on both sides of the head, face, trunk, spine, or extremities. This method was used in the first prospective, double-blinded control trial of STC technology in assessing the hemihypohidrosis/hemianhidrosis of Horner's Syndrome created by unilateral stellate ganglion blockade $(35,36)$.

\section{Stage I Linear Gradient Assessment}

There are conditions in which STC values are higher or lower distally or proximally along the axis of an extremity. In addition, there are disorders which are associated with increases in conductivity at intermediate levels along the extremity, with well defined proximal and distal boundaries. The standard method used for the evaluation of either of these phenomena consists of several sequential measurements made along a predetermined longitudinal or transverse path. Since one of the main goals of this technique is to detect the anatomic level at which the greatest rate of change of measured values occurs, it was originally named the Type C linear gradient method. Since the body surface is irregular, variations in the axis of measurement are not truly rectilinear; therefore, some workers prefer to use the shorter term, gradient montage, as an alternate name for this technique.

\section{Stage II Peak/Perimeter Determination}

The earliest clinical application of STC technology was directed toward the localization of peaks or troughs of sudomotor activity within a predetermined larger territory of relative hypohidrosis, euhidrosis, or even hyperhidrosis. This method was performed by making several measurements along various axes specifically to determine the points at which zones of relatively high STC levels would drop. This type of measurement was repeated along vertical, horizontal, and diagonal axes until the perimeter of the abnormally high STC region had been defined. By repeating measurements within that territory, the site of maximum or peak STC could be determined, and interpreted clinically, relative to the spatial distribution of the patient's symptoms or signs under various conditions (34-37).

\section{Stage III Comprehensive Sympathetic} Sudomotor Assessment: The Patel Grid

This technique involves measuring sympathetic skin activity levels at an equal number of sites along horizontal and vertical axes. After theoretical and analytical consideration, Patel was able to determine the optimum number of measurements acceptable for clinical practice to be 36 (i.e. 6 rows and 6 columns), the center of each point of measurement to be located either 1 or 2 electrode diameters away from the next adjacent measurement site.

All measurements are divided into quadrants, beginning to the right of the midline. The same sequence is followed for each of the standardized procedures noted below (S01-S17). Since the measurements are made in the same order, this system has a generally universal applicability to most regions of the body.

\section{Current Procedures}

At present, most STC procedures used for the assessment of regional sympathetic sudomotor function related to complex pain syndromes or structural lesions consist of either standardized (S01-S17) Patel Grid montages designed for each body region, or the stage II Peak/ Perimeter technique. Neither of these methods completely replaces the linear gradient montage (S18), which is often a close second choice to the peak/perimeter method for establishing the boundaries of pain-related hypohidrotic or hyperhidrotic regions.

\section{Standardization of Selective Tissue Conductance Methods II: Quantitative Analysis of Sympathetic SUDOMOTOR DATA}

Inherent within the established computer measurement systems for STC evaluation of sympathetic sudomotor function are several analytical approaches. The advantage of multiple mathematical analyses is the objective confirmation of the presence and general topographic distribution of abnormally low or abnormally high values over the region being tested.

\section{Asymmetry Ratio}

This mathematical expression was originally considered to be the most simple and practical way to detect an asymmetry in the electrophysiologic values measured during clinical sympathetic sudomotor testing. It consisted of a two step process in which each homologous data pair was first inspected to determine which measurement was larger. The second step was to divide the larger value by the smaller, and express the result as a ratio. Any ratio value greater than 1.5:1 was considered to be abnormal, and the higher the ratio, the more severe was the asymmetry.

Inherent within this method, however, was an error which occurred whenever one of the values was too low to be measured by the STC device, such as one might find when measuring sudomotor function over a region of true anhidrosis or marked hypohidrosis. Stated simply, any result less than $1 \mathrm{nS} / \mathrm{cm}^{2}$ would be displayed as a zero (0). During analysis of homologous data pairs, any value equal to, or greater than, 1 would then be divided by zero, which would equal infinity, a result which would be unusable for clinical interpretation. 
There are at least three possible ways to correct this error. The first would be to add the number 1 to all values in the measurement series. The second would be to use instead the Asymmetry Index, and the third would be to use the Ratio of Individual to Mean Values to be described in the following two paragraphs.

\section{Asymmetry Index}

One alternate method by which asymmetric data may be expressed numerically is the Asymmetry Index proposed by Woodley in 1982. This technique compared the values measured from one site of each homologous pair with the sum of values obtained at both sites of the pair, for example, $L /(L+R)$.

\section{Ratio of Individual to Mean Values}

This method of quantitative analysis has been in use since 2001 as an extension of the multiple site measurement system known as the Patel Grid.

The first step in the analysis is to obtain the mean value of all 36 samples, after which each individual value is divided by the mean and expressed as a ratio. If the value thus obtained is less than 1.5 , or greater than 0.5 , the ratio is considered to be within the normal range for that sample. The greater the deviation away from the mean, the more severe is the abnormality. The only disadvantage to this method is that extreme individual values can contribute more to the mean, in the same way that occurs when using an average reference electrode in clinical electroencephalography. Therefore it is used only rarely in clinical practice.

\section{Two Dimensional Display and Analysis}

Current standards of practice indicate that the assessment of regional sympathetic sudomotor function should be made at multiple homologous sites on both sides of the body. Therefore the STC levels and the ratio of individual to mean values are displayed in a manner consistent with the spatial distribution of the body regions from whence the data were measured. The application of this basic four quadrant display mode gains increased clinical relevance when compared to the various types of regional abnormalities of sudomotor function outlined in the following sections.

\section{Standardization of Selective Tissue Conductance Methods III: Classification of Regional Sympathetic Sudomotor Dysfunction}

\section{General Categories of STC Abnormality}

Abnormalities of sympathetic sudomotor function are most often described in terms of a) their spatial distribution, and $b$ ) the direction of the deviation of values away from the normal (i.e. increased or decreased).

The four general categories used to indicate the regional distribution of sudomotor dysfunction are based upon the degree of abnormalities present in each of the four quadrants of the Patel Grid. These patterns are labeled Focal, Unilateral, Bilateral, and Diffuse, with subcategories provided only for common variations of the abnormality.

Each group is further subdivided relative to abnormally low function (hypohidrosis / anhidrosis) or abnormally high function (hyperhidrosis).

The interpretation of low sudomotor activity has been related to the loss of function of sympathetic efferent paths to the affected region, often due to non-irritative, surgical, destructive (ablative) lesions, or regional anesthesiologic (neurolytic) blockade.

Conversely, high sudomotor activity has been related to increased excitation of postganglionic sudomotor efferent activity created by direct irritation in the sympathetic chain ganglia. Direct activation notwithstanding, regional hyperhidrosis can also be evoked indirectly: e.g. the electrodermal changes that are elicited through the increased efferent sympathetic activation initiated by noxious afferent sensory input, similar to mechanisms related to complex regional pain syndrome Type I/RSD, Type II/causalgia, and sympathetically maintained pain (SMP).

Clinical reports of sudomotor abnormalities that occur in many forms of illness have contributed significantly to our ability to classify sympathetic hypofunction or hyperfunction based on topographic distribution. As a result of this type of clinicopathological correlation with electrophysiological data obtained from STC assessment it has been possible to formulate the Classification of Regional Sympathetic Sudomotor Dysfunction as presented in Table 1 .

\section{Focal Sudomotor Dysfunction}

\section{A. Focal Hypohidrosis / Anhidrosis}

Focal hypohidrosis is a term indicating a very local or well circumscribed territory of skin in which the normal functions of sweating have been reduced or destroyed. In its more severe form (i.e., when no sweating can occur at all) the correct classification is focal anhidrosis. The focus, or area of special attention, is often small; as one might find in a healed skin lesion or scar. When the distal portion of a cutaneous nerve is transected surgically, or blocked with a regional anesthetic agent, the area of hypohidrosis or anhidrosis can still fall within one field of examination using the Patel Grid.

\section{Clinical Case Presentation: Focal Hypohidrosis / Anhidrosis}

A 20-year-old apprentice sheet metal worker sustained superficial lacerations of the left hand in a work related injury. He was transported to an emergency department where the examining physician felt that since the patient was able to move all fingers generally well, the major nerves, tendons, and muscles had been spared. Two separate 1.5 centimeter cuts were identified over the distal third of the hypothenar region, one on each of the palmar and dorsal surfaces. Two weeks after the accident, the patient continued to demonstrate normal hand and finger movements, but complained about lost or reduced sensation to cold, light touch, and pin, in a partial band around the hand. Four weeks later the skin had healed fully, but the patchy area of reduced cutaneous sensation remained. Standard methods for recording median and ulnar motor nerve conduction velocities as well as median, ulnar, and radial sensory nerve action potentials evoked by digital nerve stimulation were normal bilaterally. In an attempt to determine if any of the autonomic nerve supply to the skin was affected, sympathetic skin assessment of sudomotor function was performed with the following results.

Application of the Selective Tissue Conductance method to the hands revealed medium STC levels over the dorsal aspect of the right hand and a mixture of medium to very high levels over the right palm. Both these findings were normal for the respective surfaces of 
Table 1. PROPOSED LONGMIRE CLASSIFICATION OF REGIONAL SYMPATHETIC SUDOMOTOR DYSFUNCTION

\begin{tabular}{|l}
\hline I. FOCAL SUDOMOTOR DYSFUNCTION \\
\hline I.A.1: Focal Hypohidrosis \\
Cutaneous skin lesion (scar) \\
Transected cutaneous nerve \\
I.A.2: Focal Hypohidrosis with Hyperhidrotic Border Zone \\
Post-Herpetic Neuralgia (PHN) \\
Meralgia Paresthetica \\
Diabetic Peripheral Neuropathy \\
Autonomic Neuropathy \\
I.B.1: Focal Hyperhidrosis \\
Complex Regional Pain Syndrome \\
Type I Reflex Sympathetic Dystrophy (RSD) \\
Type II Causalgia \\
Sympathetically Maintained Pain (SMP)
\end{tabular}

I.B.2. Focal Hyperhidrosis with Contralateral (Mirror) Image Complex Regional Pain Syndrome

Type I Reflex Sympathetic Dystrophy (RSD)

Type II Causalgia

Sympathetically Maintained Pain (SMP)

I.B.3. Focal Hyperhidrosis with Multiple Foci of Increased Sudomotor Levels

Fibromyalgia Syndrome

Myofascial Pain Syndrome

\section{UNILATERAL SUDOMOTOR DYSFUNCTION}

II.A: Unilateral Hypohidrosis (Hemihypohidrosis / Hemianhidrosis)

Migraine with aura

Horner's Syndrome

Stellate ganglion blockade

Apical lesion of the lung

Brain stem infarction

Unilateral cerebral infarction

II.B.1. Unilateral Hyperhidrosis: (Hemihyperhidrosis, Primary, Ipsilateral)

Cluster headache

Irritative stellate ganglion lesion

Irritative brainstem lesion

II.B.2: Unilateral Hyperhidrosis (Hemihyperhidrosis, Secondary, Contralateral)

Stellate ganglion blockade

Unilateral sympathectomy

II.C: Unilateral Hypohidrosis with Contralateral Hyperhidrosis

Unilateral ablative brainstem lesion

Unilateral cerebral hemispheric infarction

Central post-stroke pain syndrome

Dejerine-Roussy Syndrome

\section{BILATERAL SUDOMOTOR DYSFUNCTION}

III.A.1: Bilateral Hypohidrosis, Symmetric

Midline cervical spinal cord lesion

Midline thoracic spinal cord lesion

Midline lumbar spinal cord lesion

Acral hypohidrosis due to anticholinergic medications

\section{III.A.2: Bilateral Hypohidrosis, Asymmetric}

Cervical radiculopathy with ipsilateral hypoesthesia

Lumbar radiculopathy with ipsilateral hypoesthesia

Cervical sympathetic block

Thoracic sympathetic block

Lumbar sympathetic block

III.B.1: Bilateral Hyperhidrosis, Symmetric

Cervical, thoracic or lumbar disc protrusion, central

Thoracic disc protrusion, central

Lumbar disc protrusion, central

Spinal stenosis

Emotional sweating

Hyperthyroidism

Acral hyperhidrosis

Idiopathic hyperhidrosis

Peripheral vasospasm (Raynaud's )

Peripheral arterial disease (PAD)

Peripheral ischemic neuropathy

Intermittent claudication

Diabetic neuropathy

Metatarsalgia

Plantar fasciitis

III.B.2: Bilateral Hyperhidrosis, Asymmetric Ipsilateral Contralateral

GI Inflammatory bowel disease

Pancreatitis

Cholecystitis

Cervical radiculopathy

Thoracic radiculopathy

Lumbar radiculopathy

Contralateral > Ipsilateral

Post cervical sympathetic block

Post thoracic sympathetic block

Post lumbar sympathetic block

\section{DIFFUSE SUDOMOTOR DYSFUNCTION}

IV.A: Diffuse Hypohidrosis

Riley-Day Syndrome

Hypothyroidism

Parkinson's Disease

Anticholinergic medications

GABAergic medications

Sympatholytic medications

IV.B: Diffuse Hyperhidrosis

Gustatory sweating

Thermoregulatory dysfunction

Hyperthyroidism

Syncope

Acute sympathetic crisis

Acute vertigo

Shock

Intense physical exercise 
the asymptomatic right hand. Relative to the left hand, the medium and high levels were interrupted by very low (anhidrotic) levels over the region of the previous injury.

Slightlylarger regions of hypohidrosis I anhidrosis can occur when more than three foci of reduced sudomotor function have coalesced or are contiguous within the area of skin being tested. As long as the regional pattern created by this type of abnormality remains within one quadrant of the Patel Grid, it is still classified as focal hypohidrosis or anhidrosis. Abnormalities of this type can be found in patients who have had surgical or anesthesiologic (neurolytic or electrical) blockade of more than one branch of a peripheral nerve, or when the postganglionic fibers from one or more of the sympathetic chain ganglia have been disrupted.

Testing for focal hypohidrosis or anhidrosis has been one of the few ways in which anesthesiologists and surgeons have been able to quantitatively test for the success of a sympathetic block or surgical sympathectomy.

\section{B. Focal Hyperhidrosis}

Focal hyperhidrosis is a term indicating a well circumscribed, local increase in sweating. Like its hypohidrosis counterpart, the size and spatial distribution of the focal hyperhidrotic area generally correlates well with those of the territory of the affected cutaneous nerve or sympathetic chain ganglion. Abnormalities of this type are most often found in patients who have sustained injuries to soft tissue or nerve trunks which have gradually developed into a complex regional pain syndrome Type I (reflex sympathetic dystrophy) or Type II (causalgia) or sympathetically maintained pain (SMP). It is most often unilateral, and generally found within one quadrant of the Patel Grid (Fig. 1). However, under rare circumstances, focal contralateral hyperhidrosis can occur as a mirror image in the homologous territory of the opposite (uninjured) limb.

Somewhat larger foci of hyperhidrosis can be created when small, well demarcated foci of increased sudomotor function have coalesced or are contiguous within the area of skin being tested. Abnormalities of this type can be found in patients who have had irritative changes or partial pressure on a slightly more proximal segment of a peripheral nerve or root. Unless the hyperhidrosis extends widely outside its quadrant of the Patel Grid, the abnormality is still considered to be focal. When the postganglionic fibers from one or more of the sympathetic chain ganglia have been compressed or otherwise irritated, it is common for what begins as focal hyperhidrosis to expand vertically into a pattern of unilateral hyperhidrosis.

Testing for regional hyperhidrosis also represents one of the few ways in which physicians have been able to localize those sympathetic abnormalities known to be part of the diagnosis of CRPS types I and II, and SMP. For example, the utility of most clinical electrodiagnostic procedures in the assessment of patients with pain has been in the ability to diagnose certain neurological disorders which may be the cause of the discomfort or suffering. It is generally understood that there are no electrodiagnostic tests to which can be used reliably measure a patient's perception of pain on a quantitative basis. Nevertheless, there has been a large body of clinical and laboratory evidence suggesting that the most accessible pathways for assessment of this type of regional neurologic dysfunction are those that subserve thermoregulatory sweating (i.e., the sympathetic sudomotor pathways).

When abnormal patterns of nerve impulse are directed along afferent pathways, abnormal sensations, including pain, may be perceived. However, when such activity is simultaneously transmitted from dorsal sensory pathways in the spinal cord, via collateral or internuncial fibers, around to the intermediolateral (IML) fasciculus, impulses are initiated in preganglionic sympathetic fibers. These, in

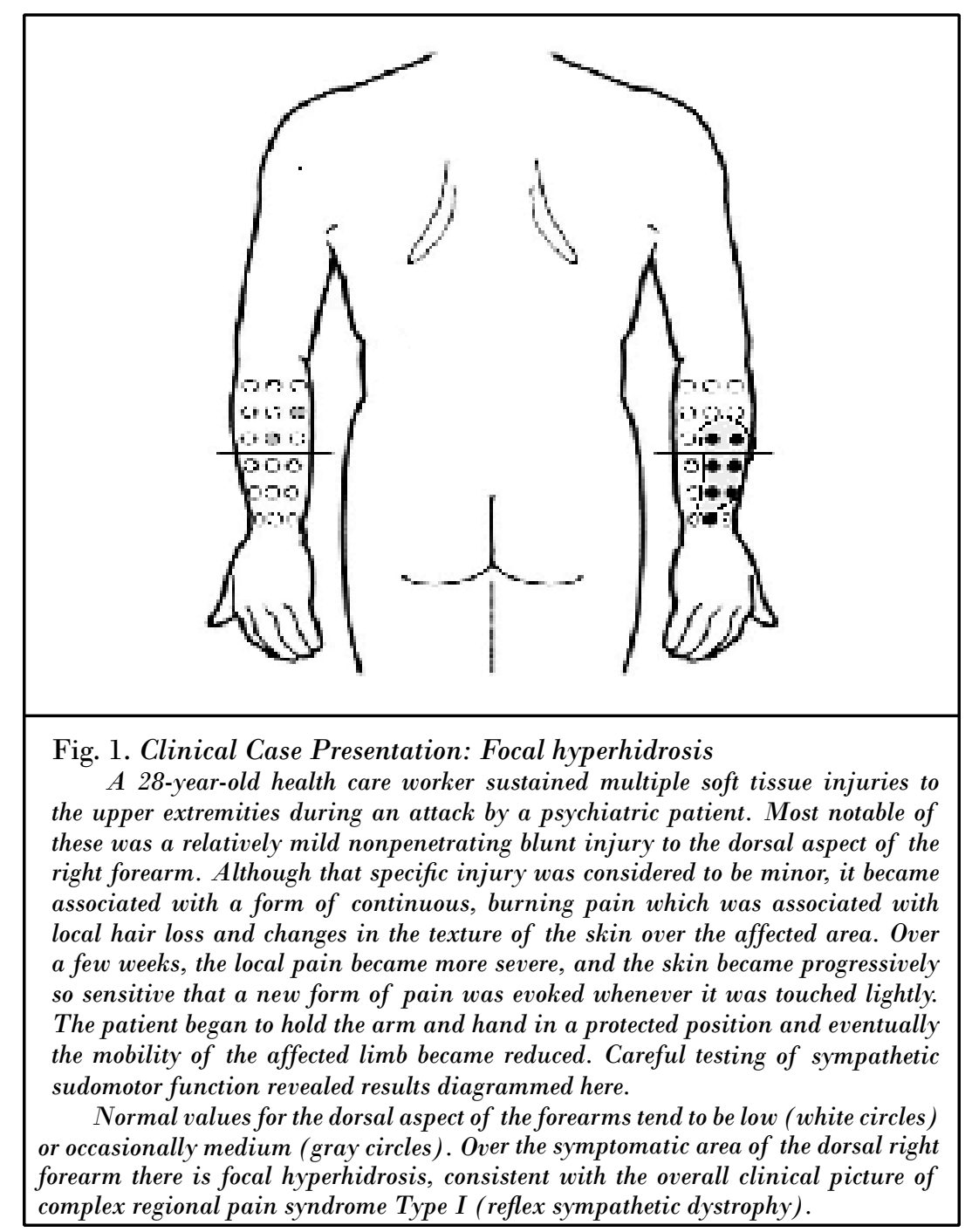


turn, stimulate neurons in the paravertebral chain ganglia, the postganglionic fibers of which ultimately end in sympathetic effectors, including sweat glands. As the nociceptive activity associated with painful tissue injury secondarily evokes higher levels of preganglionic activity in the IML, subsequent neural firing in the postganglionic supply to the skin results in local sweating in the painful area. Because of the columnar organization of these pathways, the increased sudomotor activity created by this pathophysiologic process is often well demarcated within the area of the original injury, or within the cutaneous territory of the affected sympathetic ganglion. In addition, the spatial distribution of the sudomotor dysfunction created by this process may be used clinically in the functional localization of several painful neuropathic disorders.

True multifocal hyperhidrosis can be found in fibromyalgia and myofascial pain syndromes in which relatively discrete areas of hyperhidrosis correspond with the location of myofascial trigger points.

\section{Focal Hypohidrosis with Hyperhidrotic Border Zones}

There are two common mechanisms which can create a well circumscribed focus of central hypohidrosis with a border zone of hyperhidrosis. The first condition is created when local hypohidrosis or anhidrosis occurs as a result of structural damage to the skin, with local scar formation. Around the edges of the scar the sudomotor effectors, like other nerve fibers, are hyperexcitable and create an interrupted rim of hyperhidrosis. This is seen commonly in patients with postherpetic neuralgia.

A second mechanism presents itself without any disruption of the skin from external lesions or trauma. Instead, the combination of an area of hypohidrosis or anhidrosis, surrounded by a rim of hyperhidrosis is created by a partial nerve injury in which the fibers subserving the central (hypohidrotic) territory are more severely damaged. In the remaining fibers in the same nerve, there is partial residual function intermixed with markedly irritated fiber activity which creates the hyperhidrosis in the surrounding tissue. The most clearcut example of this occurs in patients with meralgia paresthetica, the sensory abnormalities of which parallel the sudomotor dysfunction, with central hypoesthesia and dysesthesia or paresthesias along the perimeter of the affected nerve territory.

\section{Unilateral Sudomotor Dysfunction}

\section{A. Hemihypohidrosis and \\ Hemianhidrosis}

Unilateral sudomotor hypofunction is currently known as hemihypohidrosis, a term suggesting a relative, rather than absolute, condition. Only when there is an absolute absence of measurable sweat on one side of the body can the term hemi-anhidrosis be correctly applied.
Hemihypohidrosis can be further subdivided into craniofacial or somatic. Conditions such as migraine with aura, cerebral hemisphere infarction, and Horner's syndrome may show ipsilateral reduction of sudomotor function over the forehead or face. Unilateral disruptive lesions of the brainstem or high spinal cord may show decreased sweat production over the same side of the body (Fig. 2).

\section{B. Hemihyperhidrosis}

Unilateral sudomotor hyperfunction is more commonly known as hemihyperhidrosis. The determination of this state is based upon the existence of an asymmetry of $>1.5: 1$ in quantitative

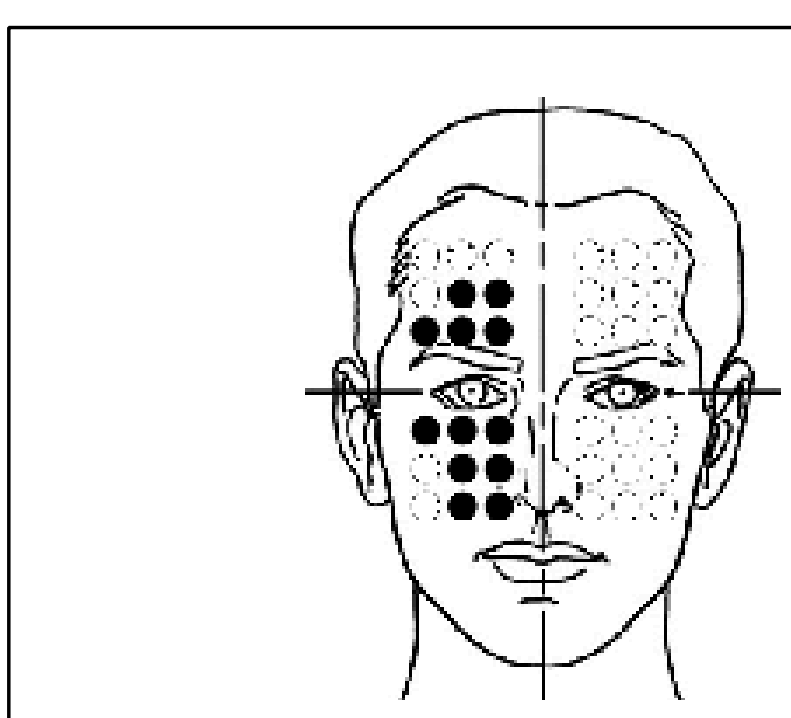

Fig. 2. Clinical Case Presentation: Unilateral Hypohidrosis/Anhidrosis with Contralateral Hyperhidrosis

A 52-year-old disabled plant foreman had developed chronic, continuous burning pain of the left upper extremity, with frequent deep pain radiating into the left neck and jaw. Associated symptoms included intermittent changes in color, temperature, and sweating of the left hand. Other than some mild scattered bilateral osteophyte formation of the cervical spine, a diagnostic workup that included EMG/NCV, MRI, CT myelography, and response to therapeutic trials of a wide range of oral analgesics, was negative. The patient was referred for anesthesiologic consultation and, after consideration of possible alternate therapies, a sympathetic neurolytic blockade of the stellate ganglion was performed. Within 3 minutes after the left stellate ganglion block, the patient developed an ipsilateral Horner's syndrome, with ptosis of the eyelid, and meiosis of the pupil. In order to determine the presence of hemi anhidrosis which would ordinarily be expected with this procedure, quantitative sympathetic sudomotor assessment was performed with the following results:

The left side of the face is anhidrotic (white circles) secondary to disruption of the sympathetic sudomotor innervation ordinarily supplied by the stellate ganglion. As a partial consequence of this hemi anhidrosis, found ipsilaterally to the nerve block, there is a secondary hyperhidrosis (black circles) at most test sites on the opposite side of the face. This combination of ipsilateral hemi anhidrosis with contralateral hemihyperhidrosis is common with neurolytic blockade of the stellate ganglia. 
sudomotor test results between the symptomatic and asymptomatic sides of the body respectively. Unlike its hypofunction counterpart, there is no upper limit to the severity of the relative symptomatic/asymptomatic ratio.

\section{Primary vs Secondary Hemihyperhidrosis}

From an etiologic viewpoint it is often convenient to classify hemihyperhidrosis as primary or secondary:

Primary hemihyperhidrosis (Class II.B.1) can be created by a single, unilateral irritative lesion. It reflects hyperactivity within those neural pathways that subserve the affected half of the body. Examples of the primary form of the disorder include cluster headache, and irritative lesions of the brainstem or lesions high in the spinal cord (Fig.3).

Secondary hemihyperhidrosis (Class II.B.2) can occur as a release phenomenon, coexisting with a contralateral (primary) hemihypohidrosis or hemianhidrosis (e.g., Horner's syndrome due to the effects of a unilateral sympathetic neurolytic block).

A very common cause of hemihyperhidrosis is created when a unilateral central irritative lesion exerts its supraspinal influence on those crossed fibers that control sudomotor function on the opposite side of the body. The most well known of this type is due to completed unilateral cerebral infarction in which the hemihyperhidrosis occurs on the same side of the body as the patient's neurological deficits, or central poststroke pain (Fig. 4).

\section{Bilateral Sudomotor Dysfunction}

\section{A. Bilateral Hypohidrosis}

Bilateral, symmetric hypohidrosis (Class III.A.1) can be found in severe peripheral diabetic, autonomic or ischemic neuropathy involving the lower extremities or in chronic post-infective or struc-

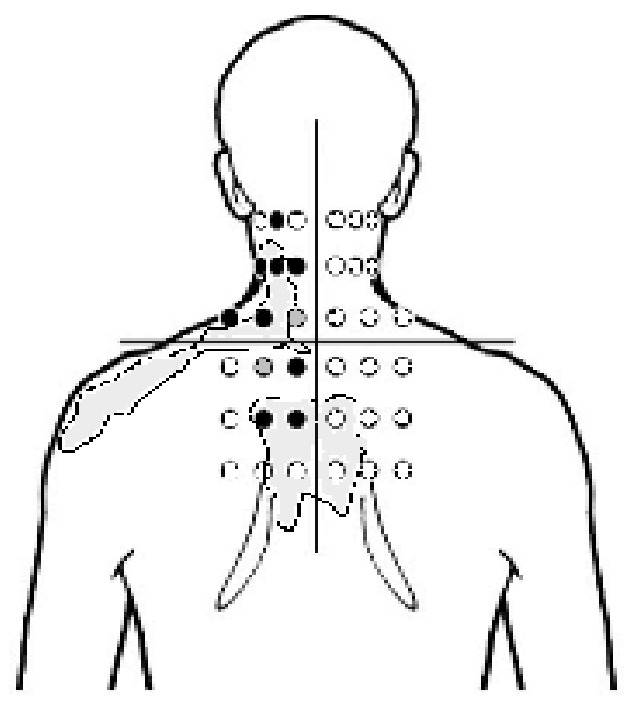

Fig. 3. Clinical Case Presentation: Unilateral Hyperhidrosis

A 46-year-old right handed female developed a fairly rapid onset of left upper extremity pain and paresthesias. These symptoms were first noted upon awakening from an uneventful sleep, and were not preceded by any trauma. The symptoms began in the left side of the neck and radiated down the anterior, posterior and lateral aspects of the arm into the hand. The quality of pain was mixed, containing both sharp pains in the neck and burning dysesthetic pains referred into the midscapular region.

High levels of sympathetic sudomotor activity (black circles) are noted over the left cervical paraspinal region and to the left of the midline near the upper portions of the medial scapular border. Spatial distribution of the cervical portion of the patient's symptoms are indicated by the shaded areas. Further diagnostic evaluation (EMG, MRI) revealed left cervical radiculopathy and protruding intervertebral dises at C4-5 and C5-6, respectively.

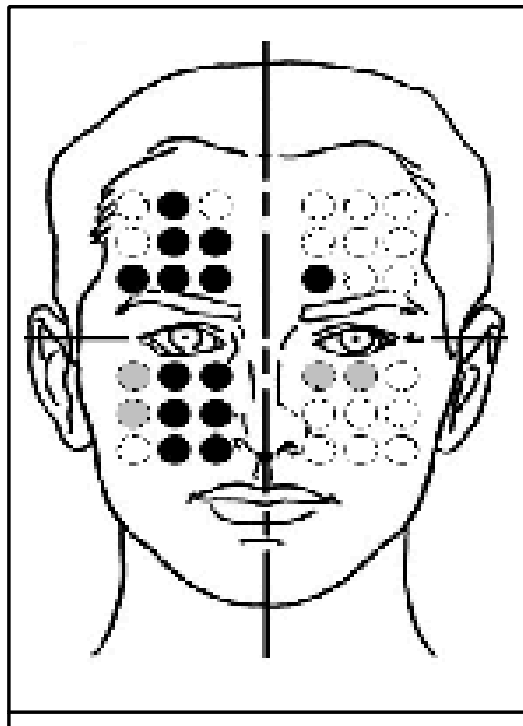

Fig. 4. Clinical Case Presentation: Unilateral Hyperhidrosis

A 64-year-old right handed hypertensive female developed right arm clumsiness and slurred speech on awakening. According to the medical history provided by her husband she attempted to get out of bed, but fell due to weakness of her right leg. Within a few minutes she had developed complete expressive aphasia (without receptive aphasia) and dense hemiplegia on the right. Early CT scan revealed no intracerebral hemorrhage, and no evidence for completed infarction. EEG revealed intermittent slow (delta) activity over the left inferior frontal/anterior temporal region with some rare sporadic sharp waves interposed. After a 5-day hospitalization, she was transferred to a rehabilitation facility for physical therapy and speech therapy. Upon admission to rehab she began to grimace, cry out, and repeatedly vocalize whenever she was touched on the affected right side. In an attempt to determine if any of the autonomic nerve supply to the skin was affected, sympathetic skin assessment of sudomotor function was performed with the following results:

It is common for sympathetic sudomotor dysfunction to be asymmetric in patients with unilateral cerebral hemisphere infarction. In more than $80 \%$ of patients monitored thus far the hemihyperhidrosis is contralateral to the ischemic lesion and ipsilateral to the patient's sensory, motor, or reflex abnormalities on clinical examination. 
tural lesions of the spinal cord, particularly those disrupting function in the anterior two thirds of the spinal cord gray matter. These are often associated with interrupted lines of mild to moderate hyperhidrosis above or below the hypohidrotic / anhidrotic zone (Fig. 5).

Bilateral, asymmetric hypohidrosis (Class III.A.2) is found more rarely than its hyperactive counterpart. It can

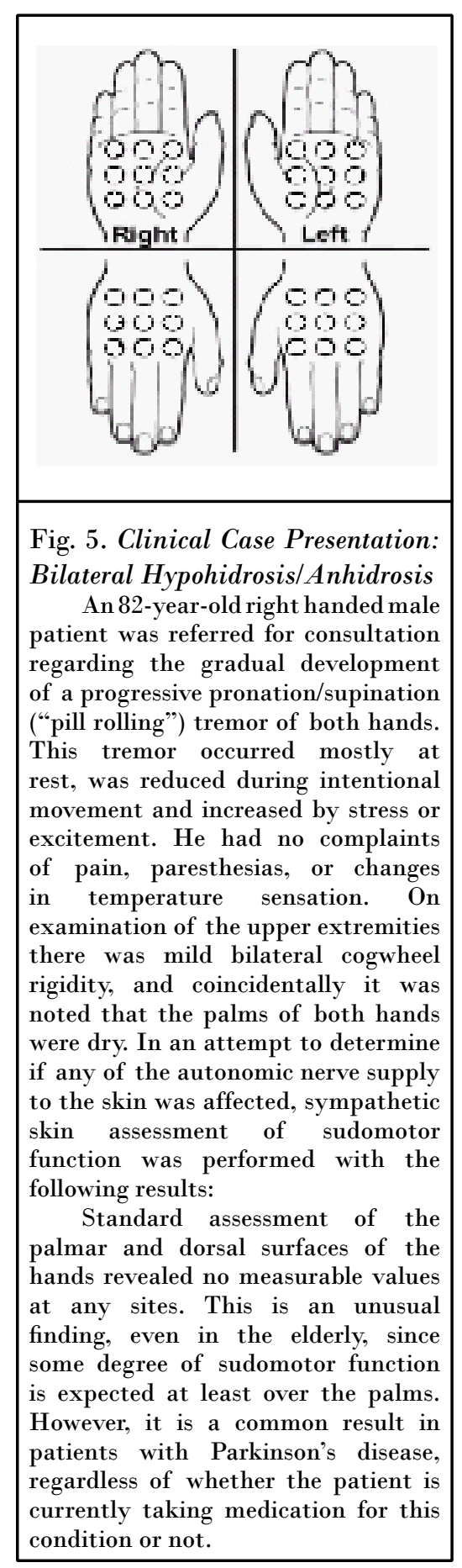

occur in patients with cervical, thoracic, and lumbar radiculopathy whose symptoms are more likely to include numbness and loss of sensation than do symptoms in those individuals who demonstrate radiculopathic pain. While most sympathetic neurolytic blocks are likely to create purely unilateral or regional hypohidrosis, there are times when the effects of cervical, thoracic, or lumbar epidural blocks create a bilateral, but often asymmetric, pattern of reduced sudomotor activity.

\section{B. Bilateral Hyperhidrosis}

Bilateral,symmetric hyperhidrosis (Class III.B.1) can be found in inflammatory or other irritative structural lesions of the spinal cord, peripheral sensory neuropathy, autonomic neuropathy or idiopathic acral hyperhidrosis.
In spinal cord lesions, this bilateral symmetric abnormality may take the form of a band of hyperhidrosis which separates euhidrotic or hypohidrotic levels above and below the affected spinal level. Another pattern related to spinal cord lesions is based upon the presentation of a band of relatively symmetric hyperhidrosis, above which the levels of sudomotor activity are low or within a range of normal. Within and below the band, however, are often found excessive levels of sympathetic skin activity which radiate distally, often into both lower extremities. More often than not, such patterns are created by symmetric compression of the thecal sac or cord by central posterior protrusion or rupture of one or more intervertebral discs with subsequent stretch of nerve roots on both sides.

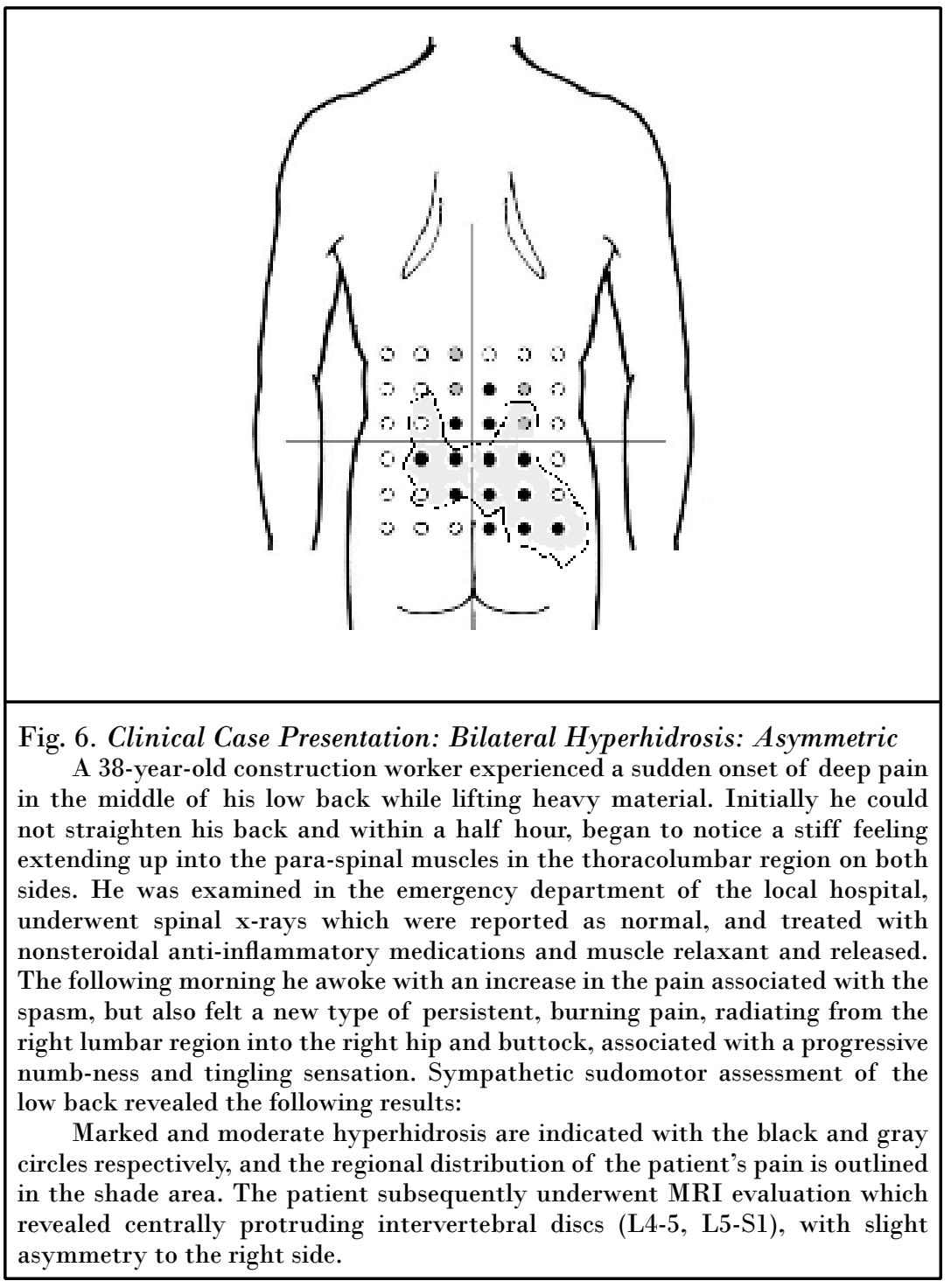




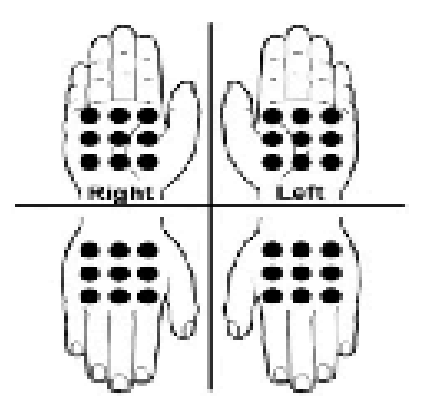

Fig. 7. Clinical Case Presentation: Diffuse Hyperhidrosis

A 33-year-old right handed female was seen in consultation for continuous fine tremor of both fingers and hands. She described herself as being a person with a lot of nervous energy. She stated that the onset of the tremor was gradual and accompanied by intermittent weakness, but without any symptoms of pain or paresthesias. However, she did state that she had weight loss of 12 pounds over 6 weeks despite an increased appetite. She also complained of marked intolerance to heat. Pertinent findings on examination revealed a slender, tremulous female with a blood pressure of $90 / 60$ and a resting pulse rate of 124 . There was mild bilateral exophthalmos but no clearcut abnormalities of the anterior neck. The forehead, face, anterior chest, hands and feet were markedly hyperhidrotic on palpation. In an attempt to determine the degree to which any of the autonomic nerve supply to the skin was affected, sympathetic skin assessment of sudomotor function was performed with the following results:

All sites on the forehead, face and palmar and hands revealed abnormally high levels of sudomotor function, even during normal room temperature. Distribution of this activity in the hands is presented here as an example: Age-adjusted normal values for the palmar surfaces ordinarily range from $15-200$, but in this patient the mean value for the left palm was 1670 , and for the right, 2200 . While the presence of diffuse hyperhidrosis does not represent a finding that is pathognomonic for the hyperthyroidism that was subsequently confirmed in this patient, it does represent an example of thermoregulatory dysfunction reflected in sympathetic sudomotor testing.
Other than central (spinal) irritation, there is a second (and more common) type of bilateral hyperhidrotic abnormality which involves the upper or lower extremities in a generally symmetric manner.

Bilateral (asymmetric, Class III.B.2) hyperhidrosis is frequently found in patients with cervical and lumbar radiculopathies (Fig. 6). Such patterns may consist of the combination of a bilateral zone of hyperhidrosis along a horizontal path approximating the appropriate spinal sympathetic sclerotomal level, with a (generally) unilateral pattern radiating into the affected limb.

\section{Diffuse Sudomotor Dysfunction}

\section{A. Diffuse Hypohidrosis / Anhidrosis}

Diffuse hypohidrosis, or even anhidrosis, is often associated with hypometabolic states and effects of medication, rather than with specific lesions of the CNS or ANS. Hypothyroidism, the effects of systemic sympatholytic medications, including anticholinergics, GABA-ergic anti-convulsants, cooling agents and general anesthesia can all cause hypohidrosis, as can certain degenerative disorders of the nervous system, such as Parkinsons disease (even in its untreated state). Most notable is the Riley-Day Syndrome in which the diffuse hypohidrosis created by congenital absence of functional sweat glands is overshadowed by almost total insensitivity to severe, and even life threatening, painful stimuli.

\section{B. Diffuse Hyperhidrosis}

Diffuse hyperhidrosis can be found as an episodic phenomenon in patients with hypermetabolic disorders such as hyperthyroidism (Fig. 7). It commonly occurs in acute conditions which evoke a marked sympathetic reaction such as vertigo, presyncope, syncope or hypovolemic shock. However, it is most often recognized as a normal physiological response to vigorous exercise or exposure to increases in environmental temperature.

\section{Standardization of Selective} Tissue Conductance Methods IV: Clinical Indications for Referral, INTERPRETATION, AND REPORTING OF DATA

\section{Clinical Indications for Referral}

Examples of the clinical disorders in which sympathetic sudomotor abnor- malities can occur have been outlined in the previous section. While these conditions may be used as examples on which to base referral for testing, the decision to request or perform electrophysiologic or other tests of sympathetic sudomotor function should also include any of the following:

1. Clinical suspicion, based upon medical history and findings on general physical examination, of autonomic nervous system dysfunction.

2. Abnormalities on neurological examination suggestive of sensory, motor, $\underline{\text { reflex}}$, autonomic or sympathetic dysfunction.

3. Changes in nonverbal behavior demonstrated in noncommunicative (aphasic, stuporous, comatose, elderly or infant) patients who have risk factors for internal disease, dysautonomia or central lesions known to cause sympathetic sudomotor abnormalities (e.g. cerebral hemispheric infarction or hemorrhage).

4. Local cutaneous abnormalities in color, temperature, hair distribution or sweating in patients with suspected complex regional pain syndrome Types I or II (reflex sympathetic dystrophy or causalgia respectively) or sympathetically maintained pain (SMP).

5. As a procedural assessment of sympathetic sudomotor abnormalities, to be performed before and after surgical sympathectomy or regional sympathetic anesthetic neurolytic blocks, or placement of neuromodulatory devices such as spinal cord stimulators or implanted programmable intrathecal drug administration systems.

Interpretation of Regional Sympathetic Sudomotor Data

The first step in the interpretation of the test results is the confirmation by the examiner that the data obtained are actually those of the patient who has undergone sympathetic sudomotor testing. Stated more succinctly, the numeric code numbers assigned to the data sheet must match those of the patient identification sheet before any interpretation is initiated.

The second step is the visual inspection, by the interpreting physician, of 
both the raw data and the results of various types of quantitative analysis. It is in this phase that the physician determines which results (e.g. mean, range, high and low absolute, high and low individual/ mean ratios) are normal or abnormal, as well as the spatial distribution of the results. It is preferred, though not always practicable, that this phase of analysis be made without referring to any of the clinical data indicating history or physical findings. This form of blind interpretation often helps to reduce bias in the clinical reporting of the data.

Next, the physician must establish the classification of abnormalities obtained during the analysis phase (i.e., I.x.x focal, II.x.x unilateral, III.x.x bilateral, or IV.x.x diffuse, and x.A.x hypohidrotic/anhidrotic, x.B.x hyperhidrotic or x.C.x mixed and X.x.1 symmetric or X.x.2 asymmetric).

The final step of analysis is the clinical correlation with the history, and physical findings already documented in earlier sections. It is in this phase of analysis that the physician must determine the severity and appropriateness of spatial distribution of the sympathetic sudomotor abnormalities as they relate to the patient's clinical data.

\section{Reporting of Data}

The final report must include all per- tinent demographic and clinical data related to the patient and the procedure: patient's name, age and gender, date of birth, identification number; date of the test procedure, address of the clinic, hospital, or laboratory where the test was performed; the name of the referring physician, and the putative diagnoses or clinical reasons for referral of the patient for the procedure.

Next, the procedure type, and standard procedure (S01-S18) code, the region of the body assessed, as well as the raw STC scores and quantitative computer analysis of data should all appear on the screen in the manner in which they will appear in the final report.

The physician will then enter the results of his or her interpretation, often beginning with the classification of the quantitative results, any clinical correlation with the patient's medical data as well as any pertinent comments. While the interpreting physician may feel obliged to suggest the potential benefits of comparing the reported STC / sympathetic data with results that might be obtained using other technical or diagnostic methods, it is essential to avoid comments that could be interpreted as recommendations for treatment. In summary, it is important to remember that this report reflects the result of a test pro- cedure, not the interpreting physician's attempt to provide a potentially well intentioned, but unsolicited, consultation or treatment plan.

\section{Conclusion}

The material presented herein has been prepared as an invited review of some current concepts and practice regarding the assessment of autonomic disorders, in particular those methods related to electrophysiological tests of sympathetic sudomotor dysfunction. Specific detail has been directed to (a) the Classification of Regional Sympathetic Sudomotor Dysfunction (CRSSD) proposed by the author and (b) the clinical laboratory evaluation of such abnormalities using electrophysiological methods such as Selective Tissue Conductance (STC) technology.

Author Affiliation:

David R. Longmire, MD

Clinical Associate Professor

Department of Internal Medicine

University of Alabama School of

Medicine-Huntsville Program

Huntsville, AL 35653

E-mail: longmire@hiwaay.net

\section{RefEREnCES}

1. American Medical Association. CPT 2005: Current Procedural Terminology. American Medical Association, Chicago, 2004.

2. Longmire DR, Stanton-Hicks M, Ranieri TA, Woodley WE, Leak WD. Laboratory methods used in the diagnosis of sudomotor dysfunction and complex regional pain syndromes: A critical review. Pain Digest 1996; 6:21-29.

3. Kuno Y. Human Perspiration. Charles C. Thomas, Springfield, IL, 1956.

4. Low PA, Caskey PE, Tuck RR. Quantitative sudomotor axon reflex tests in normal and neuropathic subjects. Ann Neurol 1983; 14:573-580.

5. Kennedy WR, Navarro X. Evaluation of sudomotor function by sweat imprint methods. In Low PA (ed.), Clinical Autonomic Disorders: Evaluation and Management. Little Brown \& Company, Boston, 1993, pp 253-261.

6. Minor L. Uber erhoten elecktrischen hautwiderstand bei traumatischen affecktionen des hals sympathies. $Z$ ges Neurol Psychiat 1923; 85:428-507.

7. Guttmann L. A demonstration of the study of sweat secretion by the quinizarin method. Proc R Soc Med 1941; 35:77-78.
8. Fealey RD. The thermoregulatory sweat test. In Low PA (ed). Clinical Autonomic Disorders: Evaluation and Management. Little Brown \& Company, Boston, 1993, pp 217-230.

9. DuBois-Reymond EH. Untersuchungen fur Thierische Electricitat, Vol 2. Reimer, Berlin,1849.

10. Brown CC. A proposed standard nomenclature for psychophysiological measures. Psychophysiology 1967; 4:260-264.

11. Veraguth P. Die Verlegung Diaskleral in das menschliche Auge einfallender Lichtreize in den Raum. Z. Psychol 1906; 42: 162-174.

12. Tarchanoff J. Décharge électriques dans la peau d l'homme sous l'excitation des organs de sens et d'activité psychiques. $C R$ Séance Soc Biol 1889; 41:447-451.

13. Tarchanoff J. Uber die galvanischen Erscheinungen an der Haut des Menschen bei Reizung der Sinnesorgane. Pflugers Arch ges Physiol 1890; 46:46.

14. Richter CP. The sweat glands studied by the electrical resistance method. Am J Physiol 1924; 68:147.

15. Lofstrom JB, Lloyd JW, Cousins MJ. Sympathetic neural blockade of upper and lower extremity. In Cousins MJ (ed.), Neural
Blockade in Clinical Anesthesia and Management of Pain. JB Lippincott Co., Philadelphia, 1980, pp 355-382.

16. Lofstrom JB, Malmquist LA, Bengtsson M. Can the sympathogalvanic reflex be used to evaluate the extent of sympathetic block in spinal analgesia? Acta Anaesthesiol Scand 1984; 28:578-582.

17. Benzon HT, Cheng SC, Avram MJ, Malloy RE. Sign of complete sympathetic blockade: Sweat test or sympathogalvanic response? Anesth Analg 1985; 64:415-419.

18. Shahani BT, Haperin JJ, Boulu P, Cohen J. Sympathetic skin response: $A$ method of assessing unmyelinated axon dysfunction in peripheral neuropathies. J Neurol Neurosurg Psychiat 1984; 47:536-542.

19. Schondorf $R$. The role of the sympathetic skin response in the assessment of autonomic function. In Low PA (ed.), Clinical Autonomic Disorders: Evaluation and Management. Little Brown \& Company, Boston, 1993, pp 231-242.

20. Vigoroux R. Sur le rôle de la résistance électrique des tissues dans l'électro-diagnostic. CR Séance Soc Biol 1879; 31:336 339.

21. Richter CP. Physiological factors involved in the electrical resistance of the skin. $A m$ J Physiol 1929; 88:596. 
22. Richter CP. Nervous control of electrical re sistance of the skin. Bull Johns Hopkins Hosp 1929; 45:56-74.

23. Richter CP. Shaw MB. Complete transactions of the spinal cord at different levels. The effect on sweating. Arch Neurol Psychiat Chicago 1930; 24:1107-1116.

24. Tower SS. Richter CP. Injury and repair within the sympathetic nervous system. I. The preganglionic neurons. Arch Neurol Psychiat Chicago 1931; 26:485-495.

25. Tower SS. Richter CP. Injury and repair within the sympathetic nervous system. II. The postganlionic neurons. Arch Neurol Psychiat Chicago 1932; 28:1139-1148.

26. Tower SS, Richter CP. Injury and repair within the sympathetic nervous system. III. Arch Neurol Psychiat Chicago 1932; 28: 1149-1152.

27. Richter CP, Levine M. Sympathectomy in man. Its effect on the electrical resistance of the skin. Arch Neurol Psychiat Chicago 1937; 38:756-760.

28. Richter CP, Katz DT. Peripheral nerve injuries determined by the electrical skin resistance method. I. Ulnar nerve. J Am Med Assoc 1943; 122:648.

29. Richter CP, Woodruff BG, Eaton BC. Hand and foot patterns of low electrical skin resistance: Their anatomical and neurological significance. J Neurophysiol 1943; 6: 417-442.

30. Richter CP, Malone PD. Peripheral nerve lesion charts. J Neurosurg 1945; 2:550-552.

31. Richter CP, Otenasek FJ. Thoracolumbar sympathectomies examined with the electrical skin resistance method. I Neurosurg 1946; 3:120-134.

32. Longmire DR, Woodley WE. Selective Tissue Conductance Meter. K874850A. Office of Device Evaluation, Food and Drug Administration, U.S. Dept. of Health and Human Services, 1988.
33. Longmire DR, Woodley WE. Selective Tissue Conductance Meter. K874850A. Office of Device Evaluation, Food and Drug Administration, U.S. Dept. of Health and Human Services, 2004.

34. Longmire DR, Woodley WE. Clinical neurophysiology of pain-related sympathetic sudomotor dysfunction. Tutorial 11. Pain Digest 1993; 3:202-209.

35. Parris WCV, Longmire DR, Lindsey K, Harrison MS. The effect of stellate ganglion block on skin conductance using Epi-Scan 5000. Presented at the $8^{\text {th }}$ Annual Meeting of the American Pain Society, Scottsdale AZ, 1989.

36. Parris WCV, Longmire DR, Harrison $M$, Prysi N. The effect of stellate ganglion block on Epi-Scan 5000 measured skin conductance. Presented at the $43^{\text {rd }}$ Postgraduate Association Meeting, New York State Society of Anesthesiologists, New York, 1989.

37. Longmire DR, Woodley WE. Selective Tissue Conductance levels in neck and shoulder pain. Presented at the 8th Annual Conference of the American Pain Society, Scottsdale, AZ, 1989.

38. Longmire DR, Parris WCV, Woodley WE, Moore C. Computerized contour mapping of Selective Tissue Conductance in sympathetically mediated craniofacial pain. Pain, Proc. VIIth World Congress of International Association for the Study of Pain, Adelaide, Australia, 1990.

39. Longmire DR, Parris WCV, Lindsey K, Woodley WE. Selective Tissue Conduc tance field plotting in patients with unilateral craniofacial pain. Presented at the $9^{\text {th }}$ Annual Conference of the American Pain Society, 1990.

40. Woodley WE, Longmire DR. Standards of practice for Selective Tissue Conductance evaluation of sympathetic sudomotor efferent functions and pain. Presented at the $10^{\text {th }}$ Annual Conference of the American Pain Society, 1991.

41. Jones EB, Longmire DR, Leak WD. Selective Tissue Conductance changes over cranial, facial and cervical regions. Presented at the $10^{\text {th }}$ Annual Conference of the American Pain Society, 1991.

42. Milner P, Leak WD, Thomas R, Longmire DR, Longmire NK. Sympathetic efferent changes affected by bolus doses of neuraxialy administered opiates. Presented at the $10^{\text {th }}$ Annual Conference of the American Pain Society, 1991.

43. Longmire DR, Leak WD, Milner P, Thomas $R$. Paradoxical increases in Selective Tissue Conductance and clinical pain related to polarity of intraspinal stimulation: Case report. Presented at the $10^{\text {th }}$ Annual Conference of the American Pain Society, 1991.

44. Leak WD, Milner P, Thomas R, Longmire DR, Longmire NK, Kennedy LD. Comparison of Selective Tissue Conductance values to patient topographic drawings. Pre sented at the $10^{\text {th }}$ Annual Conference of the American Pain Society, 1991.

45. Longmire DR, Parris WCV. Selective Tissue Conductance in the assessment of sympathetically mediated pain. In Parris WCV (ed.) Contemporary Issues in Chronic Pain. Kluw er Academic, Boston, 1991, pp 147-160.

46. Leak WD, Longmire DR, McDonald RT. Reduction in cervical pain intensity and craniofacial sudomotor activity during treatment with gabapentin. Proceedings of the VIII World Congress of Pain, International Association for the Study of Pain, Vancouver, Canada, 1996.

47. Longmire DR, Woodley WE, McDonald RT, Leak WD. Decreases in craniofacial Selective Tissue Conductance in patients obtaining pain relief with gabapentin. Proceedings of the VIII World Congress of Pain, International Association for the Study of Pain, Vancouver, Canada, 1996. 